\title{
Kernos
}

Revue internationale et pluridisciplinaire de religion grecque antique

$10 \mid 1997$

Varia

\section{Chr. AELLEN, À la recherche de l'ordre cosmique}

\section{Marie-Christine Villanueva Puig}

\section{(2) OpenEdition}

\section{Journals}

Édition électronique

URL : http://journals.openedition.org/kernos/672

DOI : $10.4000 /$ kernos. 672

ISSN : 2034-7871

Éditeur

Centre international d'étude de la religion grecque antique

Édition imprimée

Date de publication : 1 janvier 1997

Pagination : 331-335

ISSN : 0776-3824

Référence électronique

Marie-Christine Villanueva Puig, "Chr. AELLEN, À la recherche de l'ordre cosmique », Kernos [En ligne],

10 | 1997, mis en ligne le 12 avril 2011, consulté le 24 septembre 2020. URL : http://

journals.openedition.org/kernos/672 ; DOI : https://doi.org/10.4000/kernos.672 


\title{
2. Comptes rendus et notices bibliographiques
}

\author{
Christian AELLEN, À la rechercbe de l'ordre cosmique. Forme et fonction \\ des personnifications dans la céramique italiote, Zürich, Akanthus Verlag, \\ 1994. 2 vol. $21 \times 27,5 \mathrm{~cm}, 284$ p., 184 pl. ISBN : 3-905083-08-6.
}

L'ouvrage de Ch. AELLEN nous invite tout simplement à un changement radical dans la manière de considérer l'iconographie de ces vases : ce n'est plus le mythe ou la légende représentés qui donnent la clé de la scène mais les Personnifications qui y participent de différentes manières, « enrichissent le noyau de la narration... permettent de souligner le message du mythe et de faire comprendre ce qui est essentiel dans l'image $»(p, 19)$. Cela dans une production dont l'A. souligne les deux niveaux d'interprétation, narratif et funéraire, avec une signification eschatologique.

Un chapitre introductif précise ces perspectives. Il justifie l'emploi du terme «Personnification» malgré ce que celui-ci peut avoir d'inexact dans le contexte antique en raison de l'usage moderne qui en est fait. Il s'agit de forces qui ont une vie propre et peuvent être considérées comme des divinités ou des demi-divinités. La difficile question de tenter de saisir si nous avons à faire plutôt à une figure divine ou au concept abstrait correspondant, à une figure purement mythologique ou déjà au fruit d'un raisonnement susceptible d'être une allégorie, traverse tout le livre, le va-etvient de l'élément ou de l'abstraction à la divinité étant considéré comme la base même de la pensée mythique.

L'A. s'explique sur la réunion de son corpus où 114 documents ont été retenus, une fois éliminées les représentations de « thèmes courants » où il regroupe les scènes funéraires, rencontres amoureuses, retours de guerrier et, ce qui aurait peut-être mérité un mot d'explication, les scènes de thiase. Il s'agit de pièces funéraires de luxe, remarquables par leur taille et leur beauté qui posent le problème du mode de réception de ces images par un public aisé fait de Grecs et d'indigènes. $80 \%$ des scènes retenues sont apuliennes et $40 \%$ appartiennent au Peintre de Darius, à ses prédécesseurs ou successeurs directs.

Ces Personnifications ont fait l'objet d'un classement en trois groupes. Au premier appartiennent celles qui ont trait à la justice cosmique, figures de la vengeance divine : la Furie, Oistros et Diké. Dans le second, l'A. regroupe celles qu'il nomme du « macrocosme » c'est-à-dire celles qui touchent au grand univers : lieux, saisons, astres, phénomènes. Enfin, viennent celles du «microcosme » ou du petit univers : les concepts abstraits qui touchent l'homme dans ses sentiments et ses rapports avec autrui comme Hypnos, Thanatos, Eunomia, Peitbo, Pothos...

Le tome 2 réunit, outre une très riche illustration, un catalogue des trois types de Personnifications classées par figure, une série d'indices et une abondante bibliographie.

Les Personnifications de l'ordre cosmique occupent une place importante dans l'ouvrage. Une première étape consiste à distinguer la Furie des Érinyes que le mythe d'Oreste fait bien connaître dans la littérature et les arts figurés attiques. La Furie italiote est au singulier. Son aspect, qui n'est pas sans évoquer celui des Érinyes, a 
cependant un aspect sauvage et farouche plus accentué : cheveux hirsutes, traits hideux et même parfois oreilles de chien ou peau noire. Son attribut caractéristique est le serpent. Elle peut aussi tenir une torche et divers armes ou instruments de chasse : épée, fouet, kentron, lagôbolon.

Quelques données chiffrées permettent une double prise de conscience : celle de l'importance de la figure dans la céramique italiote et la manière dont sa présence infléchit la signification d'une scène mythique. Les Furies apparaissent dans 47 épisodes différents. Toutes les représentations italiotes de ces épisodes se chiffrent à 220 documents et la Furie intervient sur un tiers d'entre eux, son moment de plus grande popularité se situant dans la $2^{\mathrm{e}}$ moitié du $\mathrm{IV}^{\mathrm{e}}$ siècle Les scènes mythologiques où elle apparaît le plus souvent sont la folie de Lycurgue, les mythes d'Ixion, de Pélops et d'Actéon ainsi que les scènes infernales. La Furie prend en main le destin du héros, elle le met en exergue tout en constituant un élément fondamental de la juste lecture du mythe. Généralement dans cette céramique funéraire il s'agit de celui d'un personnage coupable et condamné. Elle se révèle ainsi comme exécutrice des desseins des dieux et souvent comme bourreau. l'A. propose de mettre en rapport sa prolifération dans des mythes très différents et son implication directe dans l'action avec certains aspects des croyances religieuses de Grande-Grèce et d'Italie du Sud où elle aurait été une figure importante, plus proche des hommes que ne l'étaient les grands dieux, impliquée dans la vie de tous les jours et la destinée en général.

Sont ensuite examinées les différentes figures relevant de la punition, de la vengeance et de la justice dans les scènes infernales, et des différences, parfois subtiles, sont établies entre la Furie, Hécate et Diké, distinguée à son tour des Érinyes. Le monde d'en-bas offrirait ainsi un système structuré de justice avec Hécate pour assurer les tâches policières, les Furies et Diké veillant à l'accomplissement des punitions requises, le tout sous l'autorité d'Hadès et en dernière instance de Zeus. "Cette justice cosmique, comme concept moral, organisée jusque dans ses moindres détails, constitue un élément fondamental dans une céramique funéraire comme la nôtre : d'une part, elle est la garantie d'un équilibre entre le bien et le mal; d'autre part, elle apporte l'espoir d'une vie après la mort, sereine et équitable. » (p. 66)

Le vase des Perses fait l'objet d'un long développement sur la valeur morale que le Peintre de Darius a voulu conférer à la scène : l'arrogante Asia, conduite par Apaté, est vouée à l'échec, tandis que la craintive Hellas est désignée par Niké pour être récompensée par les dieux.

D'intéressantes remarques concernent la complexité de la fonction temporelle de la Furie : selon son emplacement dans la scène et la direction de son regard, elle ne se rapporte pas nécessairement à l'instant présent mais peut indiquer que le héros se trouve à un tournant décisif de sa vie, où sa destinée va changer radicalement passant du pire au meilleur comme Andromède ou du meilleur au pire comme CEdipe ou Méléagre. Cet élargissement temporel du récit peut contribuer au changement d'éclairage porté sur un mythe par rapport à celui de l'imagerie attique.

Enfin, est posée la question de la Furie comme Personnification, figure concrète ou illustration d'un concept abstrait. Plusieurs critères sont retenus : son caractère visible pour le héros, signe de sa nature concrète, son indépendance vis-à-vis des sentiments de celui-ci qui la confirme comme figure autonome. Mais le problème est complexe et sur le vase des Perses par exemple, on pourrait analyser Apaté comme transcrivant une modification du comportement d'Asia, ce qui se passe à l'intérieur du 
personnage donc, changement qui d'ailleurs produit des répercussions sur l'entourage. La Furie peut encore représenter un état pathologique comme en témoigne une image d'Hippolyte. Aussi la question figure concrète ou entité abstraite reste-t-elle sans réponse tranchée. C'est dans la coexistence des deux aspects qu'est vue la richesse de ces figures.

Un deuxième temps de l'étude est consacré aux Personnifications du macrocosme, celles du temps, des phénomènes naturels, des lieux ou encore des produits agricoles, à celles du moins qui jouent un rôle cosmique. Celles qui assument une fonction proprement mythologique comme Acheloos lors du combat contre Héraklès ou Éos avec Céphale ne sont pas retenues. Entre la représentation directe d'un indice topographique et la figure mythologique protagoniste d'un mythe, se situent les Personnifications ou forces naturelles développées en figures anthropomorphes mais qui restent comme rattachées à leur élément : Crèté assise sur un rocher qui symbolise son île par exemple. Faut-il suivre jusque dans son ultime conclusion la subtile analyse de l'A. qui distingue, sur une loutrophore du Peintre de Darius où est représenté le mythe de Niobé, le cas exceptionnel d'une double présence de l'héroïne sur l'image dans sa fonction mythologique et sous sa forme cosmique : une mère affligée en voie de pétrification et la première représentation d'une montagne dans l'art grec, identifiée par une inscription comme le Sipyle mais, selon l'A., figure féminine et orientale qui désigne plus précisément le rocher de Niobé, l'héroïne une fois métamorphosée, dans la chaîne asiatique du Sipyle.

De manière générale, les Personnifications italiotes du macrocosme se distinguent de leurs homologues attiques par une spécification plus marquée grâce à leur nom inscrit, leurs attributs et leurs attitudes qui à la fois clarifient et intensifient leur rôle dans l'image. Les nymphes des lieux, les Personnifications des saisons sont successivement examinées. Le vase des Perses fait encore l'objet d'un développement particulier : Asia et Hellas y sont à la fois des réalités géographiques et politiques. Elles figurent parmi les divinités où elles transposent ce qui se passe sur le plan humain : l'attitude hautaine d'Asia est le reflet de l'bybris du Grand Roi, faute tragique qui doit être vengée. Le peintre évoque simultanément les causes et les conséquences de cette erreur et place l'image au niveau moral, ce qui interdit selon l'A. de voir en Asia, comme cela a été parfois proposé, l'image de l'Ionie, des cités grecques révoltées contre l'oppression perse.

Outre la localisation dans l'espace, les personnalités cosmiques assurent aux mythes un ordre temporel et sur ce point leur apport est particulièrement riche car on assiste comme à un « éclatement temporel» sous l'influence de ces personnifications qui enrichissent la narration par des allusions à des épisodes antérieurs ou postérieurs. Ainsi, sur un cratère sicilien, Maron remet à Ulysse l'outre de vin entre les figures d'Ampélis et d'Opora qui tiennent respectivement une corne à boire et une couronne, renvoyant selon l'A. à un au-delà de la scène strictement évoquée, à un exploit futur du héros, l'enivrement et l'aveuglement de Polyphème. Le chapitre se conclut sur un essai d'évaluation de l'activité de ces Personnifications vis-à-vis des dieux et des hommes, de leur aspect de médiateur entre les deux sphères, de leur rôle enfin par rapport au spectateur lorsque leur présence ne se contente pas de localiser ou de situer dans le temps l'épisode mais vient en modifier la signification profonde et situer son message en terme d'allégorie funéraire. 
La troisième et dernière partic, plus brève, est consacrée aux Personnifications du microcosme, c'est-à-dire celles qui renvoient à un univers plus personnel de l'homme, celui de ses émotions et de ses relations sociales. Personnifications de l'éthos, elles mettent l'accent sur l'atmosphère générale de la scène. Ainsi Euphémia, le silence religieux dans la scène du sacrifice de Phrixos, lorsque le bélier est déjà là et le salut imminent, polarise l'attention et symbolise l'ambiance religieuse du moment. Sur une scène de mariage d'Héraklès et d'Hébé, ce sont Eunomia et Eutbymia qui donnent comme la clé de lecture de l'image : l'une se situe plutôt du côté d'Apollon, qui assiste aussi à l'événement, et garantit l'ordre et la mesure, l'autre est plus liée à Dionysos, également présent, et à l'allégresse de la fête. Les deux concepts à la fois s'opposent et se complètent, et c'est sur cette polarité qu'est mis l'accent dans ces noces.

L'ultime interrogation porte à nouveau sur le passage d'une figure mythologique concrète à une entité conceptuelle abstraite. Existe-t-il vraiment des Personnifications dans la céramique italiote? Question qui reste sans réponse tranchée même si plusieurs critères sont retenus : la dynamique de la figure, son lien avec le sujet mythologique, l'inscription qui l'accompagne (on note un intéressant développement sur l'écriture dans l'image), sa réalité cultuelle, un critère de ressemblance enfin, présenté comme une hypothèse de travail : si une figure ressemble à une autre clairement dénotée comme divinité, le peintre l'aurait sans doute ressentie comme personnalité mythologique concrète. Mais le partage est très malaisé entre celles qui tiennent beaucoup du daimon et celles qui sont plus proches d'abstractions et l'A. propose finalement que la seule Personnification du corpus soit peut-être celle d'Euphémia où l'artiste est passé du concept à la figure pour visualiser une ambiance, celle du silence religieux.

La conclusion réaffirme la manière dont, dans les scènes mythologiques de la céramique italiote, les Personnifications sont devenues des figures-clés auxquelles est confié un message funéraire et comment elles peuvent modifier les données d'un mythe pour placer une scène dans une atmosphère eschatologique. Une loutrophore apulienne de Malibu très complexe où est représentée Léda est considérée comme emblématique et réunirait quatre types de personnifications : aux côtés de l'héroïne dont s'approche le cygne, Hypnos est là pour altérer sa conscience. Au registre supérieur où siègent les dieux, on note la présence d'Astrapé, le foudre de Zeus et surtout celle d'Éleusis et d'Eniautos. Signifient-ils que la défunte, représentée sur l'autre face du vase et identifiée à l'héroïne, était initiée et par conséquent qu'elle est victorieuse de la mort ? La sirène, présente sur le col de la loutrophore, pourrait symboliser, sur le plan mythologique, l'union de l'héröne avec le dieu et, sur le plan profane, l'envol de l'âme de la défunte. Enfin, la tête féminine placée de l'autre côté du col serait comme la «personnification » de l'immortalité bienheureuse.

Le problème de l'utilisation des mythes dits «scabreux » comme ceux de Médée, Actéon ou Penthée, dans une telle perspective se pose. Il s'agit alors, selon l'A., de la dénonciation d'un danger. L'accent est mis sur un individu coupable pour lequel un châtiment s'impose afin de rétablir l'ordre perdu. Enfin, l'œuvre se termine sur une réflexion liant la valeur abstraite ou concrète des figures au problème du nom inscrit à leur côté et du lien qui s'établit entre les deux pour conclure à des figures ambivalentes : entités concrètes et notions abstraites complémentaires.

L'immense intérêt de cet ouvrage très riche réside dans la nouveauté du regard porté sur ces Personnifications qui peuplent les scènes de la céramique italiote. La décision d'ensemble de faire porter sur elles l'intérêt, jointe à de fines et subtiles 
analyses, le plus souvent convaincantes, rend ainsi intelligibles des scènes difficiles où la présence de telle figure restait incompréhensible ou dont le sens général échappait. Sans doute, le parti choisi de diviser ces figures en trois groupes, d'ailleurs très inégaux en nombre comme en intérêt, conduit-il à quelques redites et donne-t-il parfois l'impression, vite dissipée, de tourner en rond. Mais surtout, l'A. prend soin de situer les Personnifications italiotes entre celles de la Grèce et celles de Rome. Il a bien notion de ce que cet art, pour neuf et original qu'il soit, doit à la littérature et aux arts figurés grecs. À maintes reprises, il fait appel au théâtre, dans sa forme écrite ou visuelle, à la poésie, à la céramique peinte de la Grèce. Néanmoins, lorsque l'analyse des Érinyes comme représentantes de la justice cosmique chez Homère apparaît à la p. 72 ou encore celle qui concerne l'organisation des puissances hésiodiques à la p. 187 dans la conclusion, on se prend à souhaiter - mais était-ce possible ? - une dimension historique et évolutive plus systématique de la réflexion sur le problème des Personnifications.

Marie-Christine VILLANUEVA PUIG (Centre Louis Gernet, CNRS, Paris)

\section{Francesco DIEz de Velasco, Los caminos de la muerte. Religión, rito e imágenes del paso al más allá en la Grecia antigua, Madrid, Ed. Trotta, 1995. 1 vol. $14,5 \times 23 \mathrm{~cm}, 198$ p. (Coll. Paradigmas). ISBN : 84-8164-016-6.}

Les lécythes à fond blanc et les textes des lamelles orphiques fournissent à l'A. une abondante documentation lui permettant de rendre compte de la multiplicité des perceptions de la mort et de l'image du chemin vers l'Au-delà. Après une introduction consacrée aux méthodes tirées de la sociologie et de l'iconographie, c'est à Ulysse « revenant», Ulysse que tous tenaient pour mort, que l'A. consacre un premier chapitre. Les deux chapitres qui suivent analysent tout d'abord les représentations iconographiques des personnages psychopompes et par là, la conception du guide sur les chemins de l'Au-delà; ce sont ensuite les différents types de représentations du mort, sous forme de corps entier, immobile, ou sous forme d'eidôlon, image désincarnée du défunt que l'A. étudie. Un long développement lui est consacré; l'A. y passe en revue les différents contextes iconographiques où apparaît l'eidôlon.

Les images des lécythes sont claires : le chemin vers l'Au-delà est certes un chemin sans retour mais les défunts morts de «belle mort » s'abandonnent sans crainte à leur guide, qu'il soit Hermès, Charon ou le couple Hypnos-Thanatos. Cette conception sereine du chemin vers l'Au-delà est née dans l'Athènes démocratique du ve siècle. Parallèlement à cela, existe une conception plus angoissante du chemin outre-tombe; celle-ci est héritée de traditions indo-européennes, mais reste marginale. C'est celle d'une mort ravisseuse, semeuse d'épouvante qu'incarnent les Harpies, les sirènes et la Gorgone. Le quatrième chapitre est une comparaison entre le tantrisme indien et les ascèses mystiques conduisant certains philosophes à tenter l'expérience de la mort; pour l'A., plusieurs récits de vie de devins grecs sont marqués par des éléments venus du tantrisme, comme la présence du serpent. Le dernier chapitre est consacré aux lamelles orphiques. C'est une conception de la mort qui est réservée au cercle restreint des initiés. Les conditions à remplir pour accomplir le voyage vers la divinité ne sont plus d'ordre social, mais se situent sur un plan spirituel: la pureté est la condition préalable pour commencer le chemin qui permet de se détacher des contingences terrestres pour d'accéder à la «vraie » vie. La mort n'est plus alors qu'un passage au terme 